\title{
ULTRASONIC ABSORPTION COEFFICIENT AND PROPAGATION VELOCITY IN CRITICAL BENZONITRILE-ISOOCTANE MIXTURE
}

\author{
T. HORNOWSKI \\ Institute of Acoustics, A. Mickiewicz University, Matejki 48/49, 60-769 Poznań, Poland
}

(Received July 13, 1992)

\begin{abstract}
Measurements of the absorption coefficient and propagation velocity of ultrasonic waves in critical benzonitrile and isooctane mixture are reported for the frequency range of 5-25 $\mathrm{MHz}$ and temperatures $\Delta T=T-T_{\mathrm{c}}$ from 0.15 to $20 \mathrm{~K}$. The analysis of the experimental results is based on dynamic scaling theory leading to good agreement with the theoretical predictions, especially in the reduced frequency range $1<\omega^{*}<100$. The adiabatic coupling constant $g$ is calculated and compared to the experimental value.
\end{abstract}

PACS numbers: $43.35 .+\mathrm{d}$

\section{Introduction}

Experimental and theoretical studies of the mechanisms underlying fast molecular processes in liquid media are now of fundamental significance for the development of molecular physics and physical chemistry. Liquid media are of special importance for the study of dynamical processes occurring in the vicinity of the critical point since due to the absence of strains, they have sharp phase transitions at their consolute temperatures $T_{\mathrm{c}}$. An excellent source of information regarding the molecular nature of the relaxation processes at the critical point is to be found in acoustic measurements, which permit the determination of quantities such as correlation length and relaxation time of the order parameter fluctuations. Moreover, they permit the extensive verification of theoretical models the importance of which reaches far beyond the scope of molecular acoustics.

Essentially, there are three theoretical approaches to the problem of acoustic wave propagation close to the critical point: the mode-coupling theory, renormalization group theory, and dynamic scaling theory. A complete discussion of the differences and similarities between the theoretical models is to be found in the papers [1,2]. More recently, research concentrates on the dynamic scaling theory due to Bhattacharjee and Ferrell (the FB theory) [3,4] who, starting from the 
thermodynamics of irreversible processes, derived the following expression for the complex compressibility of a critical mixture:

$$
\tilde{\beta}_{S}(\omega)=\beta_{S}^{c}-\frac{g^{2}}{T \rho \tilde{c}_{P}(\omega)},
$$

where $\beta_{S}^{c}$ is the adiabatic compressibility in the critical point, $\rho$ - the density of the mixture, $\tilde{c}_{p}(\omega)$ - complex frequency dependent specific heat at constant pressure, and $g$ - the adiabatic coupling constant, relating the amplitude of the oscillations in temperature and the amplitude of the oscillations in pressure of the acoustic wave. The numerical value of $g$, characteristic for a given mixture, provides a measure of the strength of ultrasonic absorption in the critical point. The $g$ is proved to be strictly noncritical as given by [5]

$$
g=-T \alpha_{P \mathrm{~b}}+\frac{T_{\mathrm{c}} \alpha_{P \mathrm{c}}}{c_{P \mathrm{c}}} c_{P \mathrm{~b}},
$$

where $\alpha_{P}$ is the thermal expansion coefficient, and $c_{P}$ is the heat capacity at constant pressure. Near $T_{\mathrm{c}}$ they can be represented by power laws of the form: $\alpha_{P}=$ $\alpha_{P \mathrm{c}} \varepsilon^{-\bar{\alpha}}+\alpha_{P \mathrm{~b}}, c_{P}=c_{P \mathrm{c}} \varepsilon^{-\bar{\alpha}}+c_{P \mathrm{~b}}\left[\varepsilon=\left(T-T_{\mathrm{c}}\right) / T_{\mathrm{c}}\right]$. Quantities with a subscript b denote the noncritical background, those with a subscript $c$ the amplitude of the critical part. Since the quantities $\rho$ and $\beta_{S}^{\mathrm{c}}$ of Eq. (1) are noncritical, the anomaly of the adiabatic compressibility $\tilde{\beta}_{S}(\omega)$ - and therefore the velocity and absorption of the wave - are attributable to $c_{P}(\omega)$ alone.

Owing to the original use of the dynamic scaling hypothesis, the FB theory has a particular merit in being capable of predicting both the thermodynamic limit (temperature dependence of zero-frequency velocity $u_{0}$ ) and the nonthermodynamic limit (frequency dependence of velocity and absorption at $T_{\mathrm{c}}$ ) - an achievement inaccessible to the mode-coupling theory because the latter starts from kinetic equations involving a critical anomaly through the equal-time correlations function. The expression for $\alpha_{\mathrm{c}} / f^{2}$ in the FB theory, where $\alpha_{\mathrm{c}} \equiv \alpha\left(T_{\mathrm{c}}\right)$ reads

$$
\frac{\alpha_{\mathrm{c}}}{f^{2}}=S f^{-(1+\bar{\alpha} / z \nu)}+\frac{\alpha(\text { back })}{f^{2}} .
$$

The constant

$$
S=\frac{\pi^{2} \bar{\alpha} c_{P \mathrm{c}} u_{\mathrm{c}} g^{2}}{2 z \nu T_{\mathrm{c}} c_{P \mathrm{~b}}^{2}}\left(\frac{\omega_{0}}{2 \pi}\right)^{\bar{\alpha} / z \nu}
$$

involves molecular parameters characterizing the critical mixture, whereas $\alpha$ (back) $/ f^{2}$ is classical absorption, related with shear viscosity and thermal conductivity. Moreover, $u_{c}$ denotes the wave velocity in the critical point, $\omega_{0}-$ the amplitude of the relaxation rate $\omega_{D}=2 D \xi^{-2}=\omega_{0} \varepsilon^{z \nu}(D-$ diffusion coefficient, $\xi$ - correlation length) associated with concentration fluctuation; $\bar{\alpha}=0.11$, $z=3.05$, and $\nu=0.63$ are critical exponents.

Bhattacharjee and Ferrell have moreover shown that $\alpha / \alpha_{\mathrm{c}}$ should scale with respect to the reduced frequency $\omega^{*}=\omega / \omega_{D}$ as follows:

$$
\frac{\alpha}{\alpha_{c}}=\frac{1}{\left[1+\left(\omega^{*}\right)^{-1 / 2}\right]^{2}}=F\left(\omega^{*}\right) .
$$


The dynamic scaling theory can also be expressed in a form involving the universal critical amplitude $A(\varepsilon)[6]$ :

where

$$
\alpha \lambda=2 \pi A(\varepsilon) G\left(\omega^{*}\right)
$$

$$
A(\varepsilon)=\frac{\pi \bar{\alpha} g^{2} c_{P \mathrm{c}} u^{2}}{4 z \nu T_{\mathrm{c}} c_{P \mathrm{~b}}^{2}} \varepsilon^{-\bar{\alpha}}=A_{0} \varepsilon^{-\bar{\alpha}}
$$

and

$$
G\left(\omega^{*}\right)=\left(\omega^{*}\right)^{-\bar{\alpha} / z \nu} F\left(\omega^{*}\right)=\frac{\left(\omega^{*}\right)^{-\bar{\alpha} / z \nu}}{\left[1+\left(\omega^{*}\right)^{-1 / 2}\right]^{2}}
$$

\section{Experimental results}

A pulse-echo method was used to measure the ultrasonic propagation velocity and absorption coefficient. For a detailed description of the measuring stand and an assessment of the accuracy obtained the reader is referred to Ref. [7].

The critical mixture benzonitrile $\left(\mathrm{C}_{6} \mathrm{H}_{5} \mathrm{CN}\right)$ and isooctane [2,2,4-trimethylpentane $\mathrm{CH}_{3} \mathrm{CHCH}_{2} \mathrm{C}\left(\mathrm{CH}_{3}\right)_{3}$ ] exhibits an upper critical point. The parameters of the separation line are [8]: $T_{c}=18.71^{\circ} \mathrm{C}$ and $x_{c}=0.465$ molar fractions of $\mathrm{C}_{6} \mathrm{H}_{5} \mathrm{CN}$. The measurements of the ultrasonic propagation velocity and absorption coefficient were carried out in the temperature range $\Delta T=T-T_{\mathrm{c}}=0.15 \mathrm{~K}$ to $20 \mathrm{~K}$ for five frequencies of the wave: $5,7,10,15$ and $21 \mathrm{MHz}$.

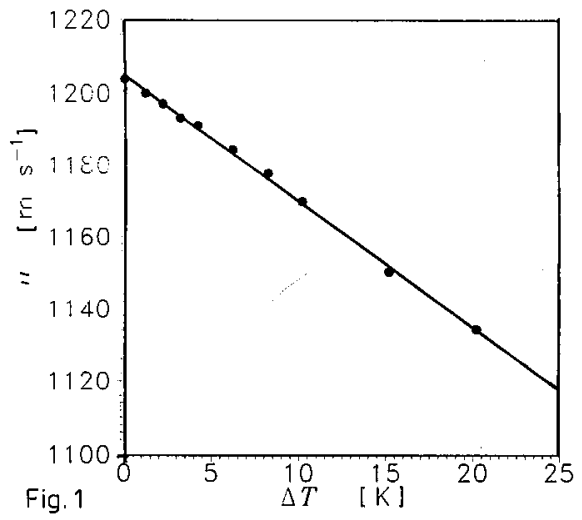

Fig. 1. Sound velocity versus temperature in critical benzonitrile-isooctane mixture at critical concentration $x_{c}=0.465$ molar fractions of $\mathrm{C}_{6} \mathrm{H}_{5} \mathrm{CN}$.

Fig. 2. Absorption $\alpha \lambda$ versus temperature in critical benzonitrile-isooctane mixture at critical concentration $x_{c}=0.465$ molar fractions of $\mathrm{C}_{6} \mathrm{H}_{5} \mathrm{CN}$.

Measurements were made in the one-phase region starting at about $313 \mathrm{~K}$ and taking successive readings as the temperature was lowered gradually down to $T_{\mathrm{c}}$. The propagation velocity $u$ is plotted versus temperature in Fig. 1. To within experimental error no velocity dispersion was found in the frequency range from 
5 to $25 \mathrm{MHz}$, whereas the dependence of the velocity versus temperature is given by the following linear plot, determined by the least squares method

$$
u=1205.04-3.50\left(T-T_{\mathrm{c}}\right) \text {. }
$$

The temperature dependence of absorption per wavelength is plotted in Fig. 2 and shows features typical for critical mixtures: a steep rise in magnitude as the critical temperature is approached, the greater the lower is the frequency of the wave.

\section{Analysis and discussion}

The observed absorption is the sum of the critical attenuation and the background (Navier-Stokes) attenuation

$$
\alpha \lambda=\alpha \lambda(\text { crit })+\alpha \lambda(\text { back }) \text {. }
$$

The background term is given by the well-known Stokes-Kirchhoff formula

$$
\alpha \lambda(\text { back })=\frac{\pi \omega}{\rho u^{2}}\left[\frac{4}{3} \eta_{\mathrm{s}}+\zeta+\Lambda\left(\frac{1}{c_{V}}-\frac{1}{c_{P}}\right)\right],
$$

where $\rho$ is the mass density, $\eta_{\mathbf{s}}$ is the shear viscosity, $\zeta$ is the noncritical bulk viscosity, and $A$ is the thermal conductivity. Unfortunately, Eq. (11) cannot be used to calculate $\alpha \lambda$ (back) since $\eta_{\mathbf{s}}$ and $\zeta$ are unknown for benzonitrile and isooctane critical mixture. However, the value of $\alpha \lambda$ (back) can be obtained by the other methods as discussed below.

As the first step in the analysis of the acoustic results let us consider the frequency dependence of absorption coefficient in the critical point. Dynamic scaling theory (Eq. (3)) predicts that $\alpha_{c} / f^{2}$ should be linear in $f^{-1.06}$, as plotted in Fig. 3, where Eq. (3) is found to give a good description of the behavior of the absorption coefficient in the critical point. The values of the coefficient $S$ and $\alpha$ (back) $/ f^{2}$ determined by the least squares method are: $S=2.68 \times 10^{-5} \mathrm{~m}^{-1} \mathrm{~s}^{0.94}$ and $\alpha$ (back) $/ f^{2}=164 \times 10^{-15} \mathrm{~m}^{-1} \mathrm{~s}^{2}$. Next, assuming that classical absorption is temperature-independent [6] one can subtract $\alpha \lambda$ (back) $=1.98 \times 10^{-10} f$ from the experimental data for all the temperatures and frequencies thus obtaining values of the absorption coefficients characterizing the relaxation process of fluctuation in concentration alone and enabling a comparison of these values with the theoretical scaling function $G\left(\omega^{*}\right)$ described by Eqs. (5) and (8). However, since data for critical variation of the diffusion coefficient $D$ and the correlation length $\xi$ are not known for benzonitrile-isooctane mixture the $\omega_{0}$ must be treated as an adjustable parameter. The results of this analysis, carried out with Bevington's CURFIT algorithm [9], are shown in Fig. 4, where the reduced absorption $\alpha \lambda$ (crit) $/ 2 \pi A$ is plotted versus $\omega^{*}$; the solid line shows the scaling function $G\left(\omega^{*}\right)$ given by Eq. (8). In the third step $\alpha \lambda$ (clas) as well as $A_{0}$ and $\omega_{0}$ were dealt as fitting parameters in CURFIT procedure. In this case a lower value of $\chi^{2}$ (value providing a measure of the fitting) was obtained. A graph of $\alpha \lambda$ (crit) $/ 2 \pi A$ versus $\omega^{*}$ for this case is given in Fig. 5.

From Figs. 4 and 5, the experimental results are found to be in quite good agreement with the theoretical scaling function proposed by Bhattacharjee and 


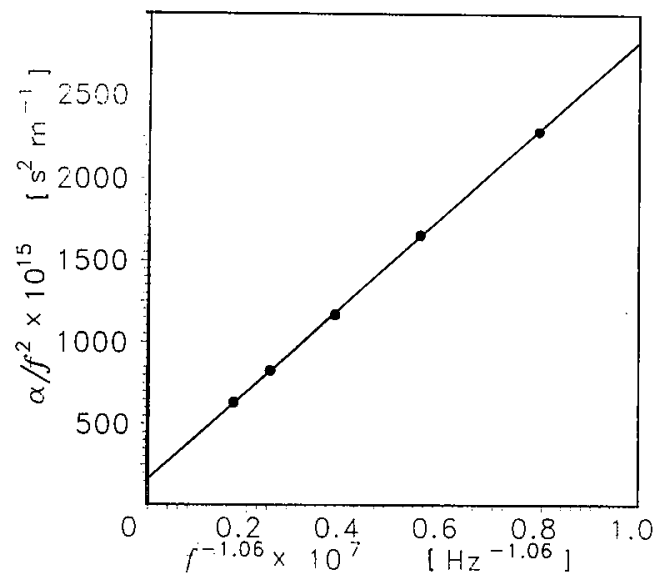

Fig. 3. Absorption $\alpha_{c} / f^{2}$ versus $f^{-1.06}$ in critical benzonitrile-isooctane mixture at critical concentration $x_{c}=0.465$ molar fractions of $\mathrm{C}_{6} \mathrm{H}_{5} \mathrm{CN}$.
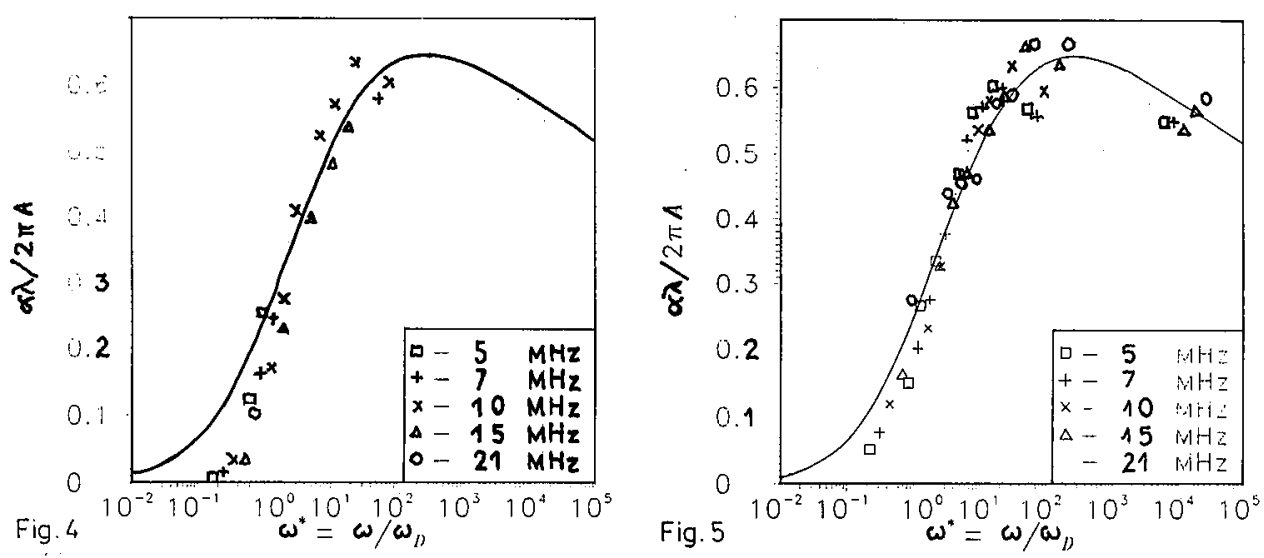

Fig. 4. $\alpha \lambda$ (crit) $/ 2 \pi A$ versus $\omega^{*}$ for five ultrasonic frequencies in benzonitrile and isooctane mixture at critical concentration $x_{c}=0.465$, the coefficients $A_{0}=1.424 \times 10^{-3}$ and $\omega_{0}=3.27 \times 10^{10} \mathrm{~Hz}$ were determined by CURFIT procedure.

Fig. 5. $\alpha \lambda\left(\right.$ crit) $/ 2 \pi A$ versus $\omega^{*}$ for five ultrasonic frequencies in benzonitrile and isooctane mixture at critical concentration $x_{\mathrm{c}}=0.465$, the coefficients $A_{0}=1.625 \times 10^{-3}$, and $\omega_{0}=2.45 \times 10^{10} \mathrm{~Hz}$, and $\alpha \lambda($ clas $)=7.8 \times 10^{-11} f$ were determined by CURFIT procedure.

Ferrell [4], especially in the range of reduced frequencies $1<\omega^{*}<100$. Certain deviations of experimental results from the scaling function $G\left(\omega^{*}\right)$ were also found by other authors [11,12]; however, at present, the amount of data is still insufficient to decide whether the function $G\left(\omega^{*}\right)$ requires some modifications. 
With the value of $A_{0}$ evaluated from acoustic measurements it is possible to determine the adiabatic coupling constant $g$. Equation (7) leads to:

$$
g^{2}=\frac{4 z \nu T_{\mathrm{c}} c_{P \mathrm{~b}}^{2}}{\pi \bar{\alpha} c_{P \mathrm{c}} u_{c}^{2}} A_{0}
$$

The density data for benzonitrile and isooctane critical mixture as a function of temperature was reported by Miller et al. [8]. From their data one can estimate the critical, $\alpha_{P \mathrm{c}}=-0.49 \times 10^{-4} \mathrm{~K}^{-1}$, and background, $\alpha_{P \mathrm{~b}}=12.48 \times 10^{-4} \mathrm{~K}^{-1}$, part of the thermal expansion coefficient. The background part of specific heat, $c_{P \mathrm{~b}}=1.95 \times 10^{3} \mathrm{~J} \mathrm{~kg}^{-1} \mathrm{~K}^{-1}$, was evaluated from the data for $c_{P}$ in the pure components whereas $c_{P \mathrm{c}}$ was calculated from the relation [8]:

$$
\frac{\mathrm{d} T_{\mathrm{c}}}{\mathrm{d} P}=\frac{T_{\mathrm{c}} \alpha_{P \mathrm{c}}}{\rho_{\mathrm{c}} c_{P \mathrm{c}}} .
$$

Taking the values of $\rho_{\mathrm{c}}=807.086 \mathrm{~kg} \mathrm{~m}^{-3}, \mathrm{~d} T_{\mathrm{c}} / \mathrm{d} P=-11.8 \times 10^{-8} \mathrm{~K} \mathrm{~Pa}^{-1}[8]$ and inserting to Eq. (13) one obtains $c_{P c}=0.15 \times 10^{3} \mathrm{~J} \mathrm{~kg}^{-1} \mathrm{~K}^{-1}$. With all necessary collateral data at hand it is possible to calculate the adiabatic coupling constant $g$ from Eq. (12). The first fit of acoustic data (Fig. $4, A_{0}=1.424 \times 10^{-3}$ ) gives $g= \pm 0.428$, whereas the second (Fig. $5, A_{0}=1.625 \times 10^{-3}$ ),$g= \pm 0.401$.

The adiabatic coupling constant can be also evaluated from thermodynamic data through Eq. (2). The value of $g$ obtained by this method equals -0.550 . The slight difference in values of $g$ can be attributed to inaccurate estimation of $c_{P \mathrm{~b}}$.

\section{Acknowledgments}

The author would like to express his gratitude to the Fulbright Committee for granting him a scholarship in the USA as well as to Prof. S. Yun at Ohio University for stimulating discussions and giving him access to the laboratory equipment for performing acoustic measurements.

\section{References}

[1] H. Tanaka, Y. Wada, Phys. Rev. A 32, 512 (1985).

[2] T. Hornowski, M. Eabowski, Acustica 72, 96 (1990).

[3] J.K. Bhattacharjee, R.A. Ferrell, Phys. Rev. A 24, 1643 (1981).

[4] R.A. Ferrell, J.K. Bhattacharjee, Phys. Rev. A 31, 1788 (1985).

[5] H. Tanaka, Y. Wada, H. Nakajima, Chem. Phys. 68, 223 (1982).

[6] C.W. Garland, G. Sanchez, J. Chem. Phys. 79, 671 (1991).

[7] T. Hornowski, M. Łabowski, Acta Phys. Pol. A79, 671 (1991).

[8] B.C. Miller, E.A. Clarke, S.C. Greer, J. Phys. Chem. 87, 1063 (1983).

[9] P.R. Bevington, Data Reduction and Error Analysis for the Physical Sciences, McGraw-Hill, New York 1969.

[10] R.A. Ferrell, J. Bhattacharjee, Phys. Rev. B 24, 4095 (1981).

[11] G. Sanchez, C.W. Garland, J. Chem. Phys. 79, 3100 (1983).

[12] 1.R. Abdelraziq, S.S. Yun, F.B. Stumpf, J. Acoust. Soc. Am. 88, 1831 (1990). 